\title{
Learning to safely approve updates to machine learning algorithms
}

\author{
Jean Feng \\ jean.feng@ucsf.edu \\ Department of Epidemiology and Biostatistics \\ University of California, San Francisco \\ USA
}

\begin{abstract}
Machine learning algorithms in healthcare have the potential to continually learn from real-world data generated during healthcare delivery and adapt to dataset shifts. As such, regulatory bodies like the US FDA have begun discussions on how to autonomously approve modifications to algorithms. Current proposals evaluate algorithmic modifications via hypothesis testing and control a definition of online approval error that only applies if the data is stationary over time, which is unlikely in practice. To this end, we investigate designing approval policies for modifications to ML algorithms in the presence of distributional shifts. Our key observation is that the approval policy most efficient at identifying and approving beneficial modifications varies across problem settings. So, rather than selecting a fixed approval policy a priori, we propose learning the best approval policy by searching over a family of approval strategies. We define a family of strategies that range in their level of optimism when approving modifications. To protect against settings where no version of the ML algorithm performs well, this family includes a pessimistic strategy that rescinds approval. We use the exponentially weighted averaging forecaster (EWAF) to learn the most appropriate strategy and derive tighter regret bounds assuming the distributional shifts are bounded. In simulation studies and empirical analyses, we find that wrapping approval strategies within EWAF is a simple yet effective approach to protect against distributional shifts without significantly slowing down approval of beneficial modifications.
\end{abstract}

\section{KEYWORDS}

Online learning; Non-stationarity; AI/ML-based SaMD; Prediction with expert advice

\section{ACM Reference Format:}

Jean Feng. 2021. Learning to safely approve updates to machine learning algorithms. In ACM Conference on Health, Inference, and Learning (ACM CHIL '21), April 8-10, 2021, Virtual Event, USA. ACM, New York, NY, USA, 10 pages. https://doi.org/10.1145/3450439.3451864

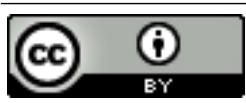

This work is licensed under a Creative Commons Attribution International 4.0 License.

ACM CHIL '21, April 8-10, 2021, Virtual Event, USA

(C) 2021 Copyright held by the owner/author(s).

ACM ISBN 978-1-4503-8359-2/21/04.

https://doi.org/10.1145/3450439.3451864

\section{INTRODUCTION}

Due to the rapid development of artificial intelligence (AI) and machine learning (ML), an increasing number of medical devices and clinical decision support software now rely on $\mathrm{AI} / \mathrm{ML}$ algorithms. The current regulatory policies of the Center of Diagnostics and Radiologic Health (CDRH) at the US Food and Drug Administration (FDA) require algorithms within AI/ML-based Software as a Medical Devices (SaMDs) to be locked, i.e. the algorithms cannot change post-approval. Nevertheless, the calibration and prediction accuracy of locked ML algorithms have been observed to degrade over time due to changes in clinical practice patterns, shifts in the patient population, and more [7, 30, 32]. Thus, there is growing interest in deploying continuously evolving ML systems that learn from data generated during healthcare delivery $[15,21,26]$ to better reflect real-world settings, adapt to distributional shifts, and incorporate new advancements in ML [25]. In anticipation of the regulatory challenges posed by such systems, the FDA plans to design processes that can automatically validate and approve modifications to ML algorithms [10,11].

The FDA's proposal in [10] calls for model developers to stipulate an Algorithm Change Protocol (ACP), which describes how algorithmic modifications will be evaluated for safety and effectiveness prior to deployment. However, this discussion document does not describe the properties of a good ACP or provide examples for how to construct one. Recent work has proposed using online hypothesis testing procedures to control the number of "bad" approvals over time [12]. This work builds on the extensive literature for testing modifications at a single time-point [5, 6, 37], paying particular attention to problems of multiple hypothesis testing and bio-creep [14]. However, a bad approval is only well-defined if the ranking between $\mathrm{ML}$ algorithms remains constant, and the error bounds in [12] assume that the data is independently and identically distributed (IID). These bounds are no longer meaningful in the presence of distributional shifts.

In this work, we propose evaluating ACPs by the cumulative risk of the approved modifications, a view commonly taken in the online learning literature. Under this framing, our aim is to answer the following question: Can we develop an ACP that controls the cumulative risk over realistic time horizons? Since the ACP operates on a regulatory level, the ACP should make no assumptions on the model development/modification process and make as few assumptions as possible regarding the data distribution. We make no structural assumptions on the type of distributional shift and even allow for adaptive distributional shifts, where the shifts can depend on which model was approved. This lets us address concerns regarding ML algorithms that induce unanticipated and 
undesirable distributional shifts, an issue that has been raised in the ML fairness literature [4, 19, 28, 29, 33].

There are a number of online learning algorithms for updating black-box ML algorithms in nonstationary settings (see e.g. the FixedShare and MarkovHedge algorithms [23, 24, 36]). However, their error bounds are too wide to be meaningful in our problem setup. Realistically, model updates are likely to occur on a quarterly basis, though one could also perform monthly or weekly updates if the data stream is rapid enough to warrant an update. Thus, our focus is on relatively few time points, say $T=50$, whereas most works in the online learning literature consider much larger values of $T$. In addition, existing methods do not control the absolute error; Instead, they control the online error relative to the best sequence of modifications, which could be arbitrarily poor. Finally, these approaches are designed for fully adversarial settings, whereas distributional shifts in real-world settings are typically smooth. In practice, these methods are overly conservative in approving modifications and outperformed by hypothesis testing methods.

In summary, our goal is to develop an ACP that i) approves beneficial modifications quickly when the data is relatively stationary and ii) protects against performance drift in the presence of smooth distributional shifts. Since the nature of the distribution shift is unknown a priori, we learn how to approve algorithmic modifications by searching across a family of approval strategies. Strategies in this family range in their level of optimism about the data distribution and include special cases like online hypothesis testing, the FixedShare algorithm, and an extremely pessimistic strategy that rescinds model approval. Using the exponentially weighted averaging forecaster (EWAF) [2], we dynamically weight the approval strategies and learn the optimal balance between safety and speed. We derive tighter error bounds assuming the distribution shifts are smooth, which significantly accelerates our ability to learn. In simulation studies and empirical analyses, we find that wrapping approval strategies within EWAF helps to protect against distribution shifts without significantly slowing down approval of beneficial modifications. Code for running L2A and reproducing this paper is available at http://github.com/jjfeng/aACP_time_trends.

\section{PROBLEM SETUP}

In this section, we describe the framework and abstractions necessary to understand the approval process for modifications to AI/ML-based SaMDs. The focus of this work is on the performance evaluation component of the ACP, which we henceforth refer to as the pACP. We note that the pACP is only one part of the ACP, which contains additional risk-mitigating procedures when deploying algorithmic updates.

We follow the approval process for algorithmic modifications outlined in [10], which depends on collecting monitoring data to track the performance of the ML algorithm and its updates over time. There are three stages in the approval cycle. First, the manufacturer proposes a modification based on available monitoring data and/or external data. This modification is added to a pool of candidate modifications and is, henceforth, locked. Second, the pACP updates the approval status of each candidate modification based on the latest monitoring data and updates the deployed algorithm accordingly. For example, a simple pACP may compare the performance of each modification to the currently deployed algorithm using a T-test and approve one with a statistically significant p-value. Third, a new batch of monitoring data is collected. We suppose this update cycle occurs on a fixed grid of $T$ time points. See Table 1 in the Supplement for a summary of the notation used in this paper.

Patient population. Let $\mathcal{P}$ be the family of joint distributions over the space of covariates $\mathcal{X}$ and range of possible outcomes $\mathcal{Y}$. Given a distribution $P \in \mathcal{P}$, patients are represented by the random vector $(X, Y)$. Let filtration $\mathcal{F}_{t}$ be the sigma algebra over all historical data-which includes the monitoring data, proposed models, and approvals by the pACP-observed up to time $t$.

Let $P_{0} \in \mathcal{P}$ be the distribution at time $t=0$. To allow for adaptive distributional shifts, we define a sequence of distribution shift functions $\left\{p_{t}: t=1, \ldots, T\right\}$, where $p_{t}$ is a $\mathcal{F}_{t-1}$-measurable function that maps onto $\mathcal{P}$. The distribution at time $t$ is the realized output from $p_{t}$, which we represent by $P_{t}$. We use the notation $P_{t_{1}: t_{2}}$ to represent the uniform mixture of (realized) distributions from time $t_{1}$ to $t_{2}$, inclusive.

At each time $t$, we observe a batch of monitoring data that contains $n$ labeled observations drawn IID from $P_{t}$. Let the empirical distribution of this batch be denoted $P_{t, n}$. Note that this assumption implies that gold-standard labels can be obtained for all observations, which is not always an easy task. For example, we exclude AI/ML-based SaMDs that predict the treatment effect, i.e. the difference in outcomes between receiving and not receiving treatment, since it is impossible to observe the counterfactual. We leave the complexities of dealing with potential outcomes to future work.

Model developer. Let $\mathcal{G}$ be a family of prediction models that map from $\mathcal{X}$ to $\tilde{\mathcal{Y}}$, where $\tilde{\mathcal{Y}}$ is the convex hull of $\mathcal{Y}$. For example, in a binary classification problem, $\tilde{\mathcal{Y}}$ is a probability between $[0,1]$. We represent modifications by an entirely new model in $\mathcal{G}$. We evaluate models using a loss function $\ell: \tilde{y} \times \mathcal{Y} \mapsto[0,1]$ that is convex with respect to the first input. The risk of a model $\tilde{g} \in \mathcal{G}$ with respect to a distribution $Q \in \mathcal{P}$ is $\mathbb{E}_{Q} \ell(\tilde{g}(X), Y)$. Its empirical risk with respect to empirical distribution $Q_{n}$ is denoted $\mathbb{E}_{Q_{n}} \ell(\tilde{g}(X), Y)$.

At each time point, the model developer can propose a new modification in an adaptive fashion. As such, we define the model development process using a sequence of functions $\left\{g_{t}: t=1, \ldots, T\right\}$, where $g_{t}$ is a $\mathcal{F}_{t-1}$-measurable function with range $\mathcal{G}$. The output from $g_{t}$ is the modification proposed at time $t$. Let the realized output be denoted $G_{t}$.

Distributional shifts. We assume that distributional shifts are bounded at each time point. Similar smoothness assumptions have been advocated in previous works [35] as a more realistic representation of real-world problem settings. We formalize this assumption in terms of the maximum mean discrepancy (MMD), a distancemeasure between probability measures [16]. For any two distributions $Q_{1}, Q_{2} \in \mathcal{P}$, define the MMD with respect to the function class $\{(x, y) \mapsto \ell(\tilde{g}(x), y): \tilde{g} \in \mathcal{G}\}$ as

$$
\operatorname{MMD}\left(Q_{1}, Q_{2}\right):=\sup _{\tilde{g} \in \mathcal{G}}\left|\mathbb{E}_{Q_{1}} \ell(\tilde{g}(X), Y)-\mathbb{E}_{Q_{2}} \ell(\tilde{g}(X), Y)\right| .
$$

So if the MMD between $P_{t}$ and $P_{t-1}$ is bounded by a constant $V>0$ for all $t=1, \ldots, T$, the maximum increase in risk for any candidate 
modification is at most $V$. More generally, suppose there is a known window size $W$ and constant $V$ such that the MMD between $P_{t}$ and distributions from the last $W$ time points are bounded follows:

Assumption 1 (Bounded MAXIMUM MEAN DISCREPANCY). There exists some constant $V>0$ and window size $W \in Z^{+}$such that the distribution shift functions $\left\{p_{1}, \ldots, p_{T}\right\}$ satisfy

$\operatorname{Pr}\left(\operatorname{MMD}\left(P_{t}, P_{\max (0, t-w): t-1}\right) \leq V \quad \forall w=1, \ldots, W\right.$ and $\left.t=1, \ldots, T\right)=1$.

Let $\mathcal{D}_{V, W}$ be all sequences of distributional shift functions that satisfy Assumption 1 for a given $V$ and $W$. In practice, the maximum shift $V$ is unknown and needs to be chosen using domain knowledge. To inform our choice of $V$, we can estimate the MMD using historical data (see e.g. [16]) to obtain a lower bound.

An abstention option. We allow the pACP to deploy prediction models with the option to abstain, also known as selective prediction models [9]. We assume there is some known cost $\delta>0$ for abstaining that is defined on the same scale as the loss. So, a selective prediction model incurs loss $\ell$ when it makes a prediction and $\delta$ when it abstains. The abstention cost should be chosen using prior knowledge; Its value should be no more than the maximum acceptable expected loss $[1,13]$.

For notational convenience, define $G_{0}$ as a selective prediction model that always abstains. In addition, define $\ell_{\delta}(z, y)$ to be the augmented loss function that is equal to $\ell(z, y)$ when $z$ is a prediction and $\delta$ when $z$ is the abstention option. Another way to view the abstention option is that the pACP has decided to temporarily rescind approval of the ML algorithm.

$p A C P$. At every time point $t$, the pACP evaluates all modifications proposed up to time $t$ using monitoring data up to time $t-1$ and updates their approval status. We generalize previous definitions of approval that only allowed "hard" approval of a single modification at each time point [12]. Here we allow for "soft" approvals as well, where each candidate modification is associated with a probability weight that reflects our confidence in its safety and effectiveness. ${ }^{1}$ More specifically, we represent the approval status by a $(t+1)$ vector from the probability simplex $\Delta^{t+1}$, where its first weight is the abstention rate and the remaining weights are associated with the candidate modifications.

We define the pACP as a sequence of functionals $\left\{\theta_{t}: t=\right.$ $1, \ldots, T\}$. To represent the information available to the pACP at each time point, define filtration $\tilde{\mathcal{F}}_{t}$ as the sigma algebra over all historical data observed up to time $t-1$ and the proposed model at time $t$. Then, $\theta_{t}$ is a $\tilde{\mathcal{F}}_{t-1}$-measurable function that maps to $\Delta^{t+1}$ Denote its realized output as $\hat{\theta}_{t}$.

Given approval status $\hat{\theta}_{t}$ at time $t$, the pACP deploys the stochastic selective prediction model $h_{\hat{\theta}_{t}}$, which abstains with probability $\hat{\theta}_{t, 0}$ and predicts using an additive ensemble over the candidate modifications with probability $1-\hat{\theta}_{t, 0}$. In particular, the ensemble is the weighted average $x \mapsto\left(\sum_{t^{\prime}=1}^{t} \hat{\theta}_{t, t^{\prime}} G_{t^{\prime}}(x)\right) /\left(\sum_{t^{\prime}=1}^{t} \hat{\theta}_{t, t^{\prime}}\right)$. The

\footnotetext{
${ }^{1}$ Soft approvals are not entirely new to the regulatory space. It shares similarities to outcome-adaptive randomization in clinical trials, which shifts the randomization ratio in favor of the more promising treatment.
}

risk of $h_{\hat{\theta}_{t}}$ at time $t$ is then

$\mathbb{E}_{P_{t}}\left[\ell_{\delta}\left(h_{\hat{\theta}_{t}}(X), Y\right)\right]=\hat{\theta}_{t, 0} \delta+\left(1-\hat{\theta}_{t, 0}\right) \mathbb{E}_{P_{t}}\left[\ell\left(\frac{\sum_{t^{\prime}=1}^{t} \hat{\theta}_{t, t^{\prime}} G_{t^{\prime}}(X)}{\sum_{t^{\prime}=1}^{t} \hat{\theta}_{t, t^{\prime}}}, Y\right)\right]$.

Over a time period of length $T$, the (realized) average risk of the $\mathrm{pACP}$ is

$$
\frac{1}{T} \sum_{t=1}^{T} \mathbb{E}_{P_{t}} \ell_{\delta}\left(h_{\hat{\theta}_{t}}(X), Y\right)
$$

For a given non-inferiority margin $\epsilon>0$, our goal is to design a pACP such that its average risk will not exceed $\delta+\epsilon$ over all distributional shift patterns in $\mathcal{D}_{V, W}$ and all adaptive model development processes. For $t=1, \ldots, T$, let $U_{t}$ be a vector of $n$ IID random variables drawn from the standard uniform distribution, which dictates how IID monitoring data is drawn from $P_{t}$. We formally express the error-rate constraint as follows:

$$
\max _{\substack{\left\{p_{t}: t=1, \ldots, T\right\} \in \mathcal{D}_{V, W} \\\left\{g_{t}: t=1, \ldots, T\right\}}} \mathbb{E}_{U_{1}, \ldots, U_{T}}\left[\frac{1}{T} \sum_{t=1}^{T} \mathbb{E}_{P_{t}} \ell_{\delta}\left(h_{\hat{\theta}_{t}}(X), Y\right)\right] \leq \delta+\epsilon .
$$

We would like to minimize (3) as much as possible as long as (4) is satisfied. We have defined this prioritization between controlling and minimizing the average risk to parallel the prioritization between Type I and II errors in hypothesis testing.

\section{LEARNING HOW TO APPROVE}

The most appropriate approval strategy for a given problem setting depends on how reliable the model development process is and how stationary the data is. This is illustrated in Figure 4 from our simulation study, which compares the cumulative risk of three fixed approval strategies across four simulation settings. In particular, we compare blind approval of algorithmic modifications, repeatedly running T-tests to identify which modifications to approve, and the MarkovHedge algorithm, which is designed for adversarial shifts. It is evident that the ranking of the approval strategies is highly variable. The MarkovHedge algorithm was the best in the presence of adaptive distributional shifts (AdaptiveShifts). The repeated T-tests were best when the shifts were small but frequent (SmallFrequentShifts) and when the data was IID but only a few modifications were beneficial (IID+RandomModels). Blind approval was best when most modifications were beneficial (IID+GoodModels).

Given such significant variation in the performance of these approval strategies, it is difficult to select a fixed one a priori. We therefore consider an approach that learns how to approve modifications. To this end, we define a family of approval strategies that vary in their prior belief about the stationarity of the data and reliability of the model development process. We then apply EWAF to learn the best strategy over time, which dynamically weights the approval strategies based on their performance. We refer to this procedure in its entirety as Learning-To-Approve (L2A).

\subsection{A family of approval strategies}

In this section, we show how approval strategies can be defined as a sequence of penalized empirical risk minimization (ERM) problems. The objective function in these ERM problems includes two 
regularization terms: one expresses our optimism in modifications proposed by the model developer, and the other expresses our optimism in our ability to predict future performance using historical data. By varying the hyperparameters associated with each regularization term, we recover a wide range of strategies that includes or approximates previously proposed strategies. Our formulation is inspired by the optimistic mirror descent algorithm [34] but considers a much wider range of "optimism."

We denote the set of all possible sequences of hard approvals up to time $T$ using $\mathcal{S}_{T}$. For convenience, suppose the hard approval sequences are ordered, so that $s_{j} \in \mathcal{S}_{T}$ denotes the $j$ th hard approval sequence. The $t$-th element of $s_{j}$, denoted $s_{j, t}$, can take on values in $\{0, \ldots, t\}$, where zero corresponds to choosing the abstention-only model $G_{0}$. To accommodate sequences of soft approvals, let $\Xi_{T}$ be the probability simplex over $\mathcal{S}_{T}$. For $\xi \in \Xi_{T}, \xi_{j}$ is the probability assigned to hard approval sequence $s_{j}$.

At every time point, the approval strategy solves a penalized ERM problem over all soft approval sequences $\Xi_{T}$. Given the solution $\hat{\xi}$ at time $t$, the approval status of modification $k$ at time $t$ is $\sum_{s_{j} \in \mathcal{S}_{T}} \hat{\xi}_{j} \mathbb{1}\left\{s_{j, t}=k\right\}$.

The objective function of the ERM problem involves two regularization terms, $\mathcal{R}_{\eta_{1}}(\xi)$ and $\xi^{\top} M_{t}$, that represent our prior beliefs about the problem setting. The function $\mathcal{R}_{\eta_{1}}: \Xi_{T} \mapsto \mathbb{R}$ for $\eta_{1} \in[0,1]$ characterizes our prior belief that modifications from the model developer are beneficial. A small $\eta_{1}$ encodes the prior belief that modifications are rarely beneficial and should be approved after thorough evaluation; a large $\eta_{1}$ encodes the prior belief that most modifications are beneficial and should be approved readily. The second term $\xi^{\top} M_{t}$ represents our prior belief that future performance can be predicted using historical data and is scaled by a penalty parameter $\eta_{2}>0$. The vector $M_{t}$ contains upper confidence bounds (UCBs) for the risk of all hard approval sequences, and the inner product $\xi^{\top} M_{t}$ is the bound for soft approval sequence $\xi$. Because the bounds assume the data is stationary, a large value of $\eta_{2}$ corresponds to the prior belief that past performance predicts future performance.

We define a family of approval strategies by varying the penalty parameters $\eta_{1}$ and $\eta_{2}$ as well as a learning rate $\eta_{3} \geq 0$. At each time point, the approval strategy for hyperparameter $\boldsymbol{\eta}=\left(\eta_{1}, \eta_{2}, \eta_{3}\right)$ solves

$$
\begin{aligned}
\min _{\xi \in \Xi_{T}} \eta_{3} & \sum_{j=1}^{\left|\mathcal{S}_{T}\right|}\left\{\xi_{j} \sum_{t^{\prime}=1}^{t-1} \mathbb{E}_{P_{t^{\prime}, n}}\left[\ell_{\delta}\left(G_{s_{j, t^{\prime}}}(X), Y\right)\right]\right\}+\eta_{2} \xi^{\top} M_{t}+\mathcal{R}_{\eta_{1}}(\xi) . \\
\text { s.t. } \xi_{j} & \leq \mathbb{1}\left\{M_{t^{\prime}, j} \leq \delta+\tilde{\epsilon}\right\} \quad \forall t^{\prime}=1, \ldots, t, \forall j=1, \ldots, t^{\prime}
\end{aligned}
$$

The objective function is the weighted average of the empirical risk across all hard approval strategies with respect to $\xi$ plus the two aforementioned regularization terms. The hyperparameters $\eta_{1}, \eta_{2}$, and $\eta_{3}$ specify the trade-off between minimizing these three terms. We refer to $\eta_{3}$ as a learning rate since it governs how much the objective function and the solution to (5) change in response to newly collected monitoring data. The constraints in (5) restrict approval to modifications with UCBs smaller than the abstention cost plus some margin $\tilde{\epsilon}$, which can be thought of as a non-inferiority margin for each time-point. We discuss how to choose $\tilde{\epsilon}$ for overall error-rate control in the next section. Note that the feasible set is

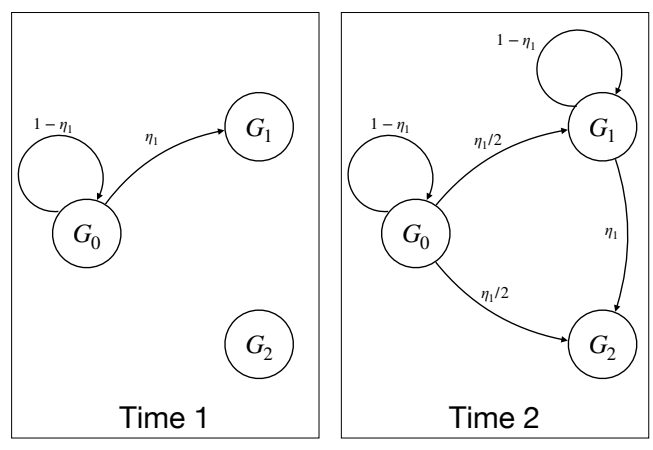

Figure 1: Example Markov chain prior over hard approval sequences for hyperparameter $\eta_{1} \in[0,1]$. Node $G_{0}$ corresponds to the abstention-only model; $G_{1}$ and $G_{2}$ are the proposed modifications at times $t=1$ and 2 , respectively. The arrows correspond to transition/approval probabilities and are labeled with transition probabilities, where an arrow returning to the current state means that nothing was approved. No arrows can enter $G_{2}$ at time $t=1$ because it is not yet available. This prior approves a new modification with probability $\eta_{1}$, only allows transitions to the current modification or later modifications, and weights all later modifications equally.

never empty because the abstention-only option always satisfies the constraints.

Approval strategies defined using (5) differ from the optimistic mirror descent algorithm $[3,34]$ in two ways. First, we scale the estimated risk bound $\xi^{\top} M_{t}$ by hyperparameter $\eta_{2}$, whereas the original formulation fixed $\eta_{2}$ to 1 . By allowing for large values of $\eta_{2}$, our family of approval strategies spans a wider range of optimism. After all, if we can predict future performance accurately, there is no need to weight models by their historical performance. Second, the added constraints disallow approval of modifications that performed poorly at previous time points. In contrast, the original formulation takes a less aggressive approach and only down-weights such models. We find that removing poor-performing modifications is more effective in practice, since model performance is usually worse at later time points, not better.

In general, solving (5) is computationally intractable since it requires searching over all possible approval sequences. Nevertheless, the approval status at time $t$ can be computed efficiently for certain choices of $\mathcal{R}_{\eta_{1}}$. We draw inspiration from the MarkovHedge algorithm [31, 36], which uses dynamic programming to efficiently search over all possible approval sequences. The MarkovHedge places a Markov chain prior over all hard approval sequences with initial distribution $a \in \Delta^{T+1}$ and transition probability matrices $A^{(2)}, \ldots, A^{(T)}$, where the accessible states at time $t$ are $G_{0}$ through $G_{t}$ (Figure 1). Let $\eta_{1}$ be the probability that a new modification is approved in this Markov prior. We show in Section B of the Supplement that the MarkovHedge algorithm corresponds to solving a 
sequence of penalized ERM problems with the regularization term

$$
\mathcal{R}_{\eta_{1}}(\xi)=\left(\sum_{j=1}^{\left|\mathcal{S}_{T}\right|} \xi_{j} \log \xi_{j}\right)-\sum_{j=1}^{\left|\mathcal{S}_{T}\right|} \xi_{j}\left(\ln a_{s_{j, 1}}+\sum_{t=2}^{T} \ln A_{s_{j, t}, s_{j, t-1}}^{(t)}\right) .
$$

We use this same definition for $\mathcal{R}_{\eta_{1}}$ in (5). The first summation in (6) is entropic regularization. The second summation is the log probability of the soft approval sequence with respect to the Markov prior, i.e. it encourages approval rates with high prior probabilities.

In Section B of the Supplement, we prove that approval statuses from strategy $\boldsymbol{\eta}$ can be computed efficiently using Algorithm 1. Rather than solving (5) directly, we use a variant of the MarkovHedge algorithm to update the approval status of the proposed modifications. In particular, Algorithm 1 looks one step ahead at each time $t$ and reweights each model $G_{j}$ based on its estimated risk bound $\tilde{M}_{t, j}$. Based on Assumption 1, we construct confidence bounds for each modification based on prospective monitoring data collected within the last $W$ time points. Thus, $\tilde{M}_{t, j}$ bounds the risk of $G_{j}$ with respect to $P_{\tau_{t, j}: t-1}$, where $\tau_{t, j}$ denotes the start index of this time window. For the special case where $j=t$, we set $\tau_{t, t}=t-1$ and construct confidence bounds using a training/validation split or cross-validation.

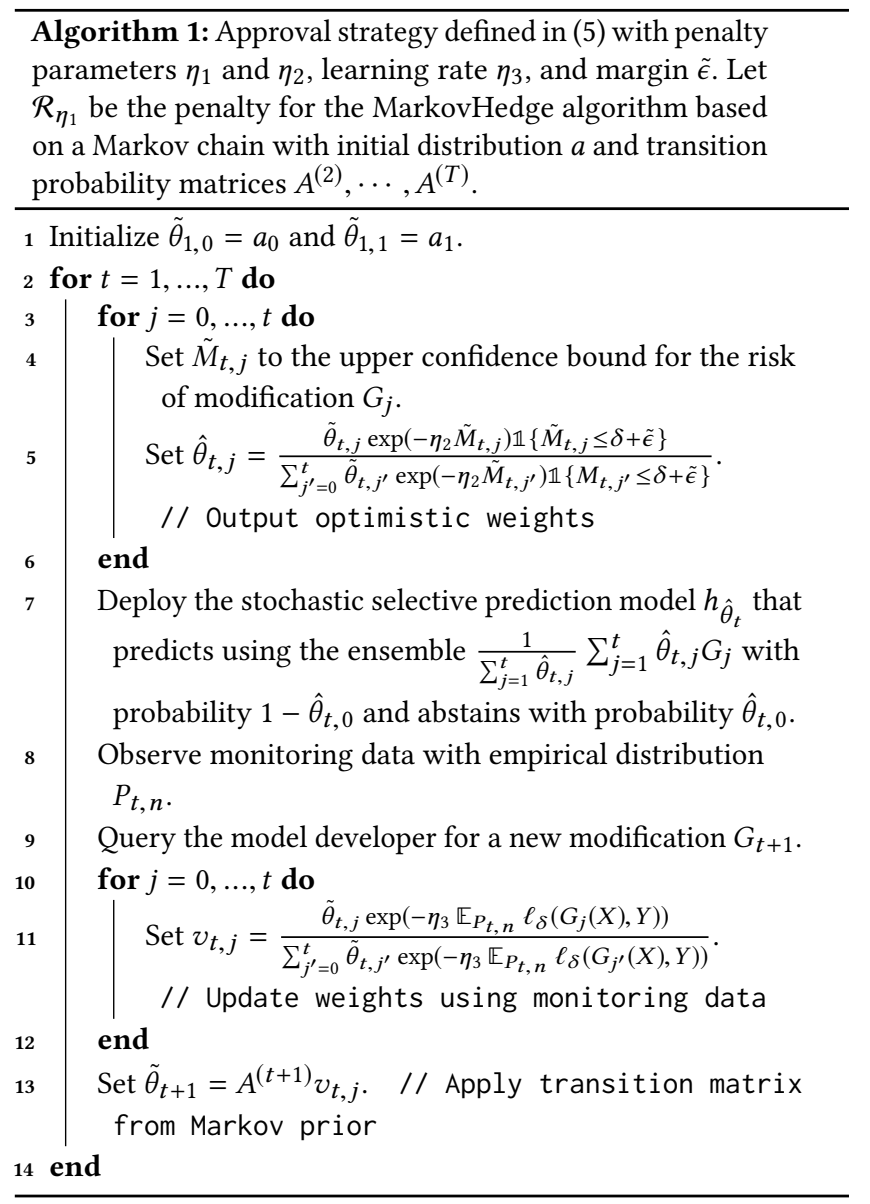

Next, we highlight approval strategies that are special cases within this family.

Abstention only. The most pessimistic strategy refuses to use any models from the developer and only abstains. This is an important special case: When distributional shifts are severe, none of the candidate modifications have acceptable performance, and the abstention-only strategy is the safest option. This corresponds to (5) with $\eta_{1}=\eta_{2}=\eta_{3}=0$. That is, the Markov chain prior assigns a probability of one to the approval sequence that uniformly abstains over time and zero to all others. This strategy ignores the empirical loss of the proposed modifications.

Blind approval. The most optimistic validation strategy approves the latest modification instantaneously. We approximate this strategy by setting $\eta_{1}$ close to one and $\eta_{2}=\eta_{3}=0$. This will approve the latest modification at each time step as long as its risk bound satisfies the constraint in (5).

Repeated T-tests against baseline. If we believe that distributional shifts are rare, an effective approval strategy is to identify modifications that are non-inferior to the abstention option by hypothesis testing and to select the one with the best historical performance. We approximate non-inferiority testing using the constraint in (5) for $\tilde{\epsilon}>0$ and select the modification with the lowest upper confidence bound by setting $\eta_{1}=0.5, \eta_{3}=0$, and $\eta_{2}$ to some large value (e.g. $>T)$.

MarkovHedge. When the distribution is highly non-stationary, we can use the MarkovHedge algorithm by setting $\eta_{1} \in(0,1)$, $\eta_{2}=0$, and $\eta_{3}>0$. At every time point, this strategy will deploy a selective prediction model with some non-zero abstention rate.

\subsection{Searching over a family of approval strategies}

L2A optimizes the approval strategy by searching over the family of strategies defined in Section 3.1 using the EWAF algorithm. Suppose we have $m$ candidate approval strategies with hyperparameters $\boldsymbol{\eta}^{(j)}$ for $j=0, \ldots, m-1$, where $\boldsymbol{\eta}^{(0)}$ is the abstention-only strategy. Let the approval status at time $t$ from strategy $\boldsymbol{\eta}^{(j)}$ be denoted $\hat{\theta}_{t}^{(j)}$. L2A searches over the candidate strategies by dynamically assigning each strategy a probability weight, where $w_{t, j}$ is the probability weight for strategy $j$ at time $t$. Initially, the probability weights for all strategies are equal. For some meta-learning rate $\lambda>0$, L2A updates the weight for the $j$ th strategy at times $t=1, \ldots, T$ as follows:

$$
w_{t, j}=\frac{w_{t-1, j} \exp \left(-\lambda \mathbb{E}_{P_{t-1, n}} \ell_{\delta}\left(h_{\hat{\theta}_{t-1}^{(j)}}(X), Y\right)\right)}{\sum_{j^{\prime}=0}^{m-1} w_{t-1, j^{\prime}} \exp \left(-\lambda \mathbb{E}_{P_{t-1, n}} \ell_{\delta}\left(h_{\hat{\theta}_{t-1}^{\left(j^{\prime}\right)}}(X), Y\right)\right)} .
$$

L2A then deploys the selective prediction model $h_{\hat{\theta}_{t}^{\mathrm{LAA}}}$ where $\hat{\theta}_{t}^{\mathrm{L} 2 \mathrm{~A}}=$ $\sum_{j=1}^{m} w_{t, j} \hat{\theta}_{t}^{(j)}$. The pseudocode for L2A is given in Algorithm 2.

Next, we bound the expected risk of L2A. Our proof, given in Section C of the Supplement, is based on existing bounds for the EWAF and improves on them in a number of ways. Because L2A has the option to abstain, we can use the abstention cost to tighten 

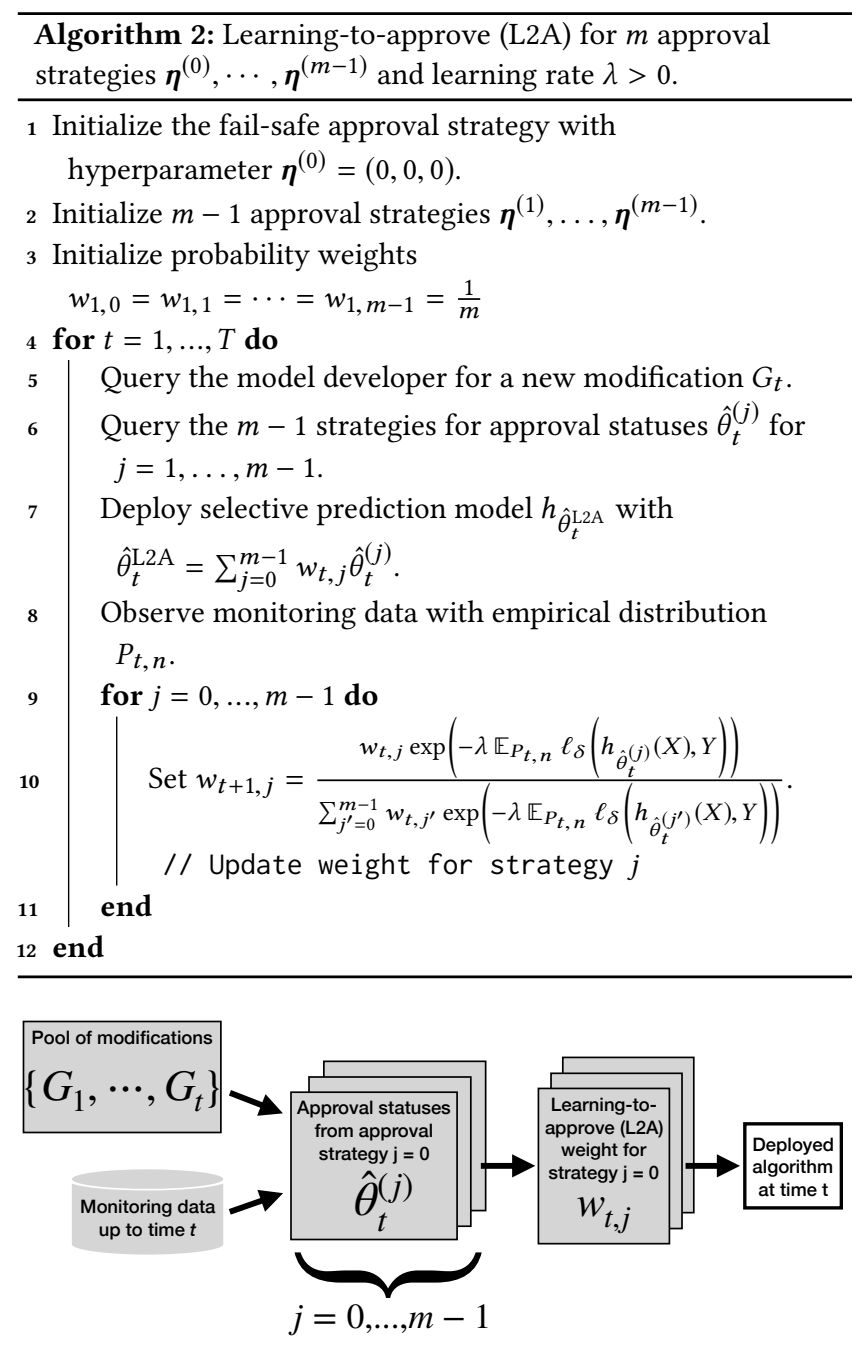

Figure 2: Illustration of learning-to-approve (L2A) with $m$ candidate approval strategies at time $t$. For $j=0, \ldots, m-1$, the $j$-th approval strategy evaluates the performance of all algorithmic modifications and outputs approval statuses $\hat{\theta}_{t}^{(j)}$. Operating as meta-algorithm, L2A evaluates the performance of the approval strategies and updates their weights $w_{t}$ accordingly. The L2A weights and approval statuses are combined to deploy a weighted ensemble at time $t$.

the risk bound. We further tighten the bounds using Assumption 1, which states that the distributional shifts are bounded. Also, we bound the risk whereas most works focus on the empirical risk. As such, we must account for sampling variability in the monitoring data. Note that the following result applies to any $T$ and can thus be used to bound the risk at intermediate time points.

THEOREM 1. Consider any sequence of distribution shift functions $\left\{p_{t}: t=1, \ldots, T\right\} \in \mathcal{D}_{V, W}$ for some $V>0$ and window size $W$, and any model development process $\left\{g_{t}: t=1, \ldots, T\right\}$. Suppose there exist constants $\alpha_{1}, \alpha_{2}, z \geq 0$ such that the following probability bounds are satisfied by the risk bounds $\left\{M_{t, j}: t=1, \ldots, T, j=\right.$ $1, \ldots, t\}$ :

$\operatorname{Pr}\left(\max _{j=1, \ldots, t} \mathbb{E}_{\mathrm{P}_{\tau_{t, j}: t-1}} \ell_{\delta}\left(G_{j}(X), Y\right)-\tilde{M}_{t, j} \geq 0\right) \leq \alpha_{1} \quad \forall t=1, \ldots, T$

$\operatorname{Pr}\left(\max _{j=1, \ldots, t} \mathbb{E}_{\mathrm{P}_{\tau_{t, j}: t-1}} \ell_{\delta}\left(G_{j}(X), Y\right)-\tilde{M}_{t, j} \geq z\right) \leq \alpha_{2} \quad \forall t=1, \ldots, T$.

Then for any learning rate $\lambda>0$ and $\tilde{\epsilon} \geq 0$, the expected average risk for L2A satisfies

$$
\begin{aligned}
& \mathbb{E}_{U_{1}, \ldots, U_{T}}\left[\frac{1}{T} \sum_{t=1}^{T} \mathbb{E}_{P_{t}} \ell_{\delta}\left(h_{\hat{\theta}_{t}^{\mathrm{LAA}}}(X), Y\right)\right] \\
\leq & -\frac{1}{c(\lambda, \tilde{\epsilon}, V, z)}\left(\lambda \delta+\frac{1}{T} \ln m+\frac{\lambda^{2}}{8 n}\right)
\end{aligned}
$$

where

$$
\begin{aligned}
c(\lambda, \tilde{\epsilon}, V, z)= & \frac{\exp (-\lambda(\delta+\tilde{\epsilon}+V))-1}{\delta+\tilde{\epsilon}+V}\left(1-\alpha_{1}-\alpha_{2}\right) \\
& +\frac{\exp (-\lambda(\delta+\tilde{\epsilon}+V+z))-1}{\delta+\tilde{\epsilon}+V+z} \alpha_{1}+(\exp (-\lambda)-1) \alpha_{2}
\end{aligned}
$$

The risk bound is small if the risk bounds $\tilde{M}_{t}$ satisfy (8) and (9) with small values of $\alpha_{1}$ and $\alpha_{2}$. We can do this by setting $\tilde{M}_{t, j}$ to the upper limit of the $\left(1-\alpha_{1} / t\right)$-confidence interval of $\mathbb{E}_{\mathrm{P}_{\tau_{t, j}: t-1}} \ell_{\delta}\left(G_{j}(X), Y\right)$, which adjusts for multiplicity using a Bonferroni correction. One may also construct tighter confidence intervals by accounting for correlations between the proposed modifications. To derive $\alpha_{2}$, we use Hoeffding's inequality. Assuming that the confidence bound for $G_{t}$ at time $t$ is constructed using a validation set of size $n^{\prime}$, then (9) is satisfied with $\alpha_{2}=(T-1) \exp \left(-8 z^{2} n\right)+\exp \left(-8 z^{2} n^{\prime}\right)$. Note that the above probability bounds only need to hold at individual time points. That is, we only need to adjust for multiplicity within time points, not across time points.

Using Theorem 1, we can choose the hyperparameters in L2A such that the average risk is controlled. (Because $z$ is only a variable that appears in the proof, we optimize its value to minimize the risk bound.) Our bound shares the same form as the classical EWAF bound of $\frac{1}{1-\exp (-\lambda)}\left(\lambda \delta+\frac{1}{T} \ln m\right.$ ) (see e.g. Theorem 2.4 of Cesa-Bianchi and Lugosi [2]) and converges at the same rate with respect to $T$. The difference is in the constants: our bound is scaled by $-1 / c(\lambda, \tilde{\epsilon}, V, z)$ and the latter is scaled by $\frac{1}{1-\exp (-\lambda)}$. As $\alpha_{1}$ and $\alpha_{2}$ converge to zero, the constant $-1 / c(\lambda, \tilde{\epsilon}, V, z)$ converges to $\frac{\delta+\tilde{\epsilon}+V}{1-\exp (-\lambda(\delta+\tilde{\epsilon}+V))}$. Thus, the upper bound scales with the maximum drift $V$ from Assumption 1.

The difference between the two results is illustrated more concretely in Figure 3, where we plot the error bounds for various abstention costs and meta-learning rates. We set $m=10, T=50$, and, for the sake of comparison, $n=\infty$. We assume the maximum drift is $V=2 \delta$. We find that the curvatures of our risk bounds are significantly flatter and allow for much larger learning rates. For example, for abstention cost $\delta=0.15$, the minimum attainable risk bound from the classical result is $2 \delta$ and corresponds to using a learning rate of $\lambda=0.70$. We can achieve this same risk bound 


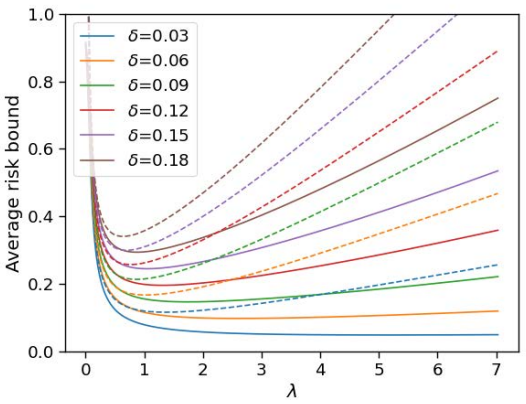

Figure 3: Comparison between risk bounds for $m=10$ and $T=50$ from Theorem 2.4 in Cesa-Bianchi and Lugosi [2] (dashed lines) and Theorem 1 (solid lines). The latter bound was derived under the assumption that the maximum distributional shift in terms of the maximum mean discrepancy is $V=2 \delta$. Each curve traces out the upper bound for different learning rates $\lambda$ values, and each color corresponds to a different $\delta$ value.

using Theorem 1 with a learning rate that is nearly three times as large, which translates to a much faster approval rate.

The upper bound in Theorem 1 grows only at a logarithmic rate with respect to the number of candidate approval strategies $m$. So for large values of $T$, we may search over a large number approval strategies with little impact on the error bound. Nevertheless, because the focus of this paper is on small values of $T$, the additional error from adding a candidate approval strategy is nonnegligible. In the following simulations and empirical analyses, we run L2A with $m$ up to 12, which give meaningful error bounds. One direction for future research is to account for similarities between the approval strategies [17] to tighten bounds and allow for more comprehensive search strategies.

\section{SIMULATION STUDY}

We now perform simulation studies to evaluate L2A's ability to control and minimize the cumulative risk. We evaluate four fixed approval strategies: abstention-only, blind approval, the MarkovHedge, and repeated T-tests. We implemented two versions of L2A, one that only searches over the four aforementioned strategies (L2A-4) and another that searches over 12 hyperparameter settings (L2A12). Additional simulation details are included in Section $D$ in the Supplement.

In the simulations below, the prediction task is binary classification, the number of time steps is $T=50$, and the sample size at each time point is $n=75$. We evaluate models using the hinge loss, a convex relaxation of the zero-one loss. At each time point, the model developer refits logistic regression on the monitoring data. We set the abstention cost $\delta$ to the risk of the initially-approved model $G_{1}$. All simulated shifts satisfy Assumption 1 with $V=\delta$ and $W=3$. This was chosen so that any model performing slightly better than the abstention option does not more than double in risk within a time window of length $W=3$. The four simulations settings are as follows:
(1) AdaptiveShifts: We simulate adaptive shifts that are targeted against the T-test approval strategy. In particular, the distribution is IID for a few time points and shifts whenever the T-test approves a new modification. In this way, the newly approved modification will perform worse than abstaining. The model is refit on data from the last four time points.

(2) SmallFrequentShifts: In many settings, distributional shifts are small but frequent. This simulation introduces small, random perturbations to the data generating mechanism every four time points. We simulate a model developer who tries to account for these shifts by varying the amount of data used to refit the data. Here, the model developer cycles between training on data from the past $j$ times points for $j=1, \ldots, 5$.

(3) IID+GoodModels: This simulation reflects the ideal problemsetting where the target population is constant over time and the proposed modifications are improving over time. We do this by refitting the model on all monitoring data up to the current time point.

(4) IID+RandomModels: Here the data is IID but the model development process is unreliable and most modifications are deleterious. To simulate this, we implement a model developer who usually refits the model only using data from the last two batches and, every four time points, refits the model on all available monitoring data.

The full list of candidate approval strategies used in L2A-12 are listed in Table 2 of the Supplement. We chose a wide range of candidate strategies that includes those highlighted in Section 3.1. At each time point, the approval strategies constructed upper confidence bounds such that (9) was satisfied with $\alpha_{1}=0.1$. We set the noninferiority margin $\epsilon$ to $0.6 \delta, \tilde{\epsilon}=0.2 \epsilon$, and used the maximum allowable learning rate according to Theorem 1 . $\lambda$ was set to 1.6 on average using this procedure.

Figure 4 shows the cumulative average risks and abstention rates for the pACPs. Notably, the ranking among the four fixed approval strategies varies across the different settings. For example, the T-test performed well in IID settings but poorly in AdaptiveShifts, and the MarkovHedge performed well in AdaptiveShifts but poorly in IID settings. Only L2A-12 performed as good as or even better than the best fixed approval strategy across all simulation studies.

L2A-12 achieved a significantly lower risk than all the other fixed strategies in SmallFrequentShifts. This is expected, because none of the four fixed strategies (or their mixtures) are optimal in this setting. By searching over a wider range of approval strategies, L2A-12 was able to identify a better strategy. In particular, L2A12 steadily converged towards the hyperparameter setting of $\boldsymbol{\eta}=$ $(0.5,100,10)$ over time.

We also investigate how the abstention rates of the different approval strategies vary over time. L2A increased the abstention rate in AdaptiveShifts, used a steady rate in SmallFrequentShifts, and decreased the abstention rate in the two IID simulations. This is the desired behavior, since the abstention option is effective for defending against distributional shifts and the proposed modifications have high predictive accuracy in stationary settings. While the MarkovHedge also learned to adjust the abstention rates over time, it learned much slower, which led to higher average risks. 

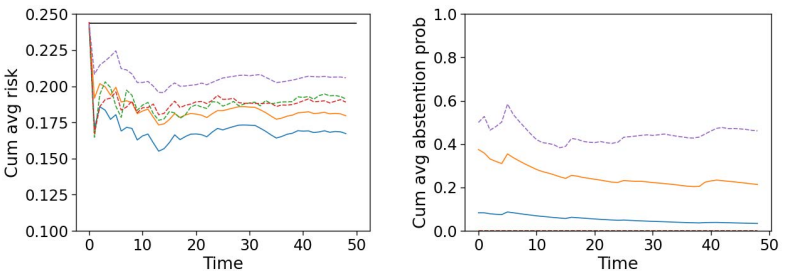

(a) AdaptiveShifts: Distribution shifts whenever the T-test approves a new modification.
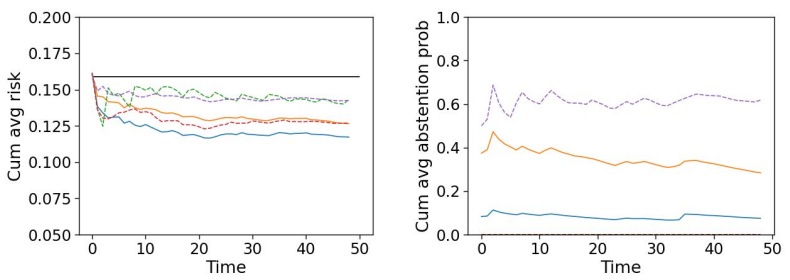

(b) SmallFrequentShifts: Distribution frequently shifts by a small amount.
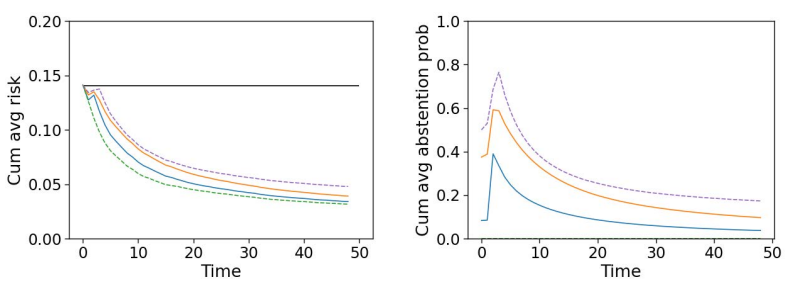

(c) IID+GoodModels: Data is IID. Most modifications are beneficial.
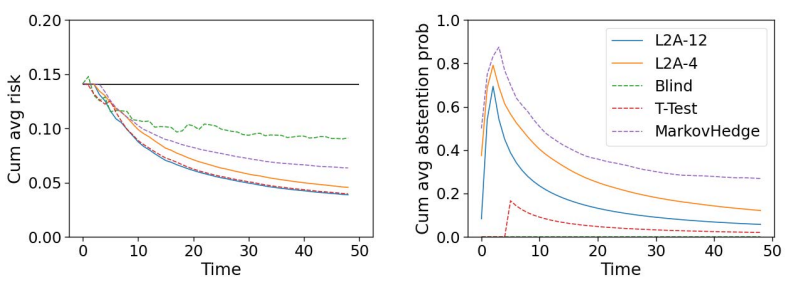

(d) IID+RandomModels: Data is IID. Only a few modifications are beneficial.

Figure 4: Simulations comparing the cumulative average risk (left) and abstention rate (right) for different approval policies. The horizontal black line corresponds to the cost of abstaining. The solid curves correspond to the Learning-toApprove algorithm with 4 and 12 approval strategies, which are labeled as L2A-4 and L2A-12, respectively. Dashed curves are fixed approval strategies: blind approval, repeated $T$ tests, and MarkovHedge. The distribution shifts over time in (a) and (b) and is IID in (c) and (d).

\section{EXPERIMENTS ON REAL-WORLD DATASETS}

In this section, we evaluate how L2A performs in the presence of real-world distributional shifts. Of course, the FDA has not yet approved continuously evolving AI/ML-based SaMDs, which means
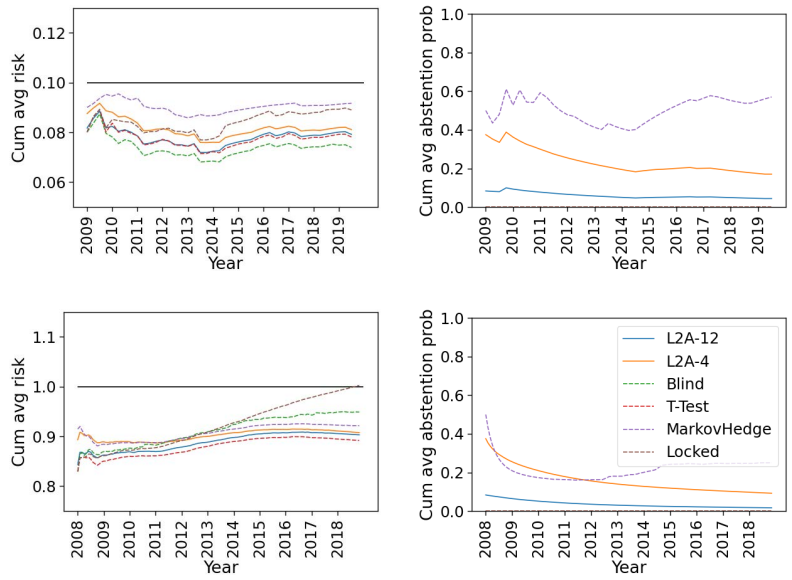

Figure 5: The cumulative average risk (left) and probability of abstaining (right) for different approval policies on the MIMIC-IV dataset (top) and the Yelp dataset (bottom). The "Locked" policy keeps the initially-approved algorithm locked.

that monitoring data for these algorithms are not available. As such, we have chosen to evaluate L2A on the Medical Information Mart for Intensive Care (MIMIC)-IV [20] and Yelp (http://www.yelp.com/ dataset) datasets, because (1) they span a time period of at least ten years and (2) every observation is associated with a timestamp. For the MIMIC-IV dataset, the data has been deidentified and the timestamps are randomly shifted within a one-year time window, which dampens existing distributional shifts. We supplement this analysis with the Yelp dataset, where the exact timestamp of each observation is available. Additional details for these analyses are given in Sections $\mathrm{E}$ and $\mathrm{F}$ of the Supplement.

\subsection{MIMIC data}

MIMIC-IV contains deidentified electronic health records for over 40,000 patients admitted to intensive care units at the Beth Israel Deaconess Medical Center between 2009 and 2019. The dates are randomly shifted to preserve patient privacy, but the approximate year is provided to let researchers study time trends in the data. Here we consider the benchmark task of predicting in-hospital mortality [18] using physiological signals, such as heart rate, pulse oximetry, and arterial blood pressure. We simulate a model developer who trains a random forest classifier and updates it on a quarterly basis by retraining on data from the past two years. We use the hinge loss to evaluate the models and set the abstention cost to $\delta=0.1$.

In Section E of the Supplement, we analyze the covariate shift in the MIMIC dataset. As shown in Figure 2 of the Supplement, a series of random forest classifiers could predict whether the data was from 2008 versus a later year with accuracy over 0.8 , which implies significant covariate shift. Nevertheless, the risk prediction model trained on the first three months of data experienced only minor performance decay: Its empirical risk increased from 0.08 to 0.1 over the ten-year time period, exceeding the abstention cost at a number of time points. This suggests that the model depended 
on relatively stable variables and/or extrapolated reasonably well. Because performance decay is slow in this problem setting, a continuously learning model has the potential to improve over time.

The performance of the different approval strategies on this dataset are shown in Figure 5 (top). Blind approval performed best in this setting, decreasing the cumulative average risk to 0.075 . However, blind approval is too risky to deploy in practice. The next best pACPs were the repeated T-tests, L2A-4, and L2A-12, which were all able to keep the average risk around 0.08 . As mentioned before, the T-test is also a risky approval strategy because it does not provide error-rate control in the presence of distributional shifts. On the other hand, as illustrated in this data analysis, L2A is able to protect against performance drift while attaining nearly the same performance as the repeated T-test. In particular, cumulative average risks of L2A-4 and the T-test converged over time, and the risk curves for L2A-12 and the T-test were essentially identical. L2A12 converged much more quickly because it searched over a larger set of approval strategies and converged quickly towards the highly optimistic approval strategies (Figure 3 in the Supplement). The MarkovHedge algorithm performed poorly on this dataset because it relied too heavily on the abstention option.

\subsection{Yelp data}

The Yelp dataset contains millions of reviews left by users from 2008 to 2018. We consider the task of predicting reviewer ratings a number between 1 and 5 - from the review text. While this task is artificial, it shares similarities to many natural language processing tasks that arise in healthcare, such as electronic phenotyping [22, $27,38]$. Here, we simulate a model developer who proposes a new deep learning model every month by training on data from the prior month. We sample 2000 reviews each month to serve as monitoring data. We define the loss using the absolute difference and set the abstention cost $\delta$ to 1 .

There is clear evidence of drift in the Yelp dataset. The average number of stars in the Yelp reviews decreased from 2008 to 2013 and then increased thereafter (Figure 1 in the Supplement). In addition, the test error of our initial model, trained only on data from January 2008, steadily increases from 0.85 to 1.15 . As shown in Figure 5 (bottom), the best approval strategy in our Yelp experiment is to repeatedly apply the T-test. However, the T-test approval strategy is not guaranteed to perform well in the presence of distributional shifts. By wrapping the T-test strategy within L2A, we can control the cumulative risk and recover similar operating characteristics. Indeed, we find that L2A-12 and L2A-4 perform almost as well as the T-test strategy in this dataset. While the fixed MarkovHedge algorithm also provides error-rate control, it is designed for adversarial setups and was overly conservative in approving modifications.

\section{DISCUSSION}

In this paper, we investigated if it is possible to design approval policies for modifications to AI/ML-based SaMDs that control the online error-rate in the presence of distributional shifts. Because distributions tend to change slowly in real-world datasets, we only assumed that the distributional shifts were bounded. Otherwise, the shifts were allowed to be adaptive, which lets us study shifts induced by the ML algorithms themselves. We observed that the most appropriate approval strategy for a given problem setting is highly dependent on how reliable the model development process is and how stationary the data is. Because this is difficult to anticipate a priori, we introduced L2A, which defines a family of approval strategies and learns the most appropriate one using the EWAF algorithm. We derive tighter regret bounds for our problem setting, which allowed us to run L2A with larger learning rates. In simulation studies and empirical analyses, we demonstrated that L2A is able to protect against adaptive distributional shifts without significantly sacrificing the rate at which beneficial modifications are approved. Our results show that wrapping existing approval strategies within L2A is a simple yet effective strategy for protecting against distributional shifts. Future work may consider more complex online learning algorithms that adapt to the stationarity (or lack thereof) in the data, such as the AdaHedge [8].

We highlight that L2A is able to control the cumulative risk in an absolute sense because it can abstain for some fixed $\operatorname{cost} \delta$. Without the abstention option, none of the pACPs considered in this paper are necessarily safe in the presence of distributional shifts. In fact, keeping the initial algorithm locked is not safe either because its performance can drift over time. Thus, the abstention option is a crucial device for controlling the error-rate when the ML algorithm is susceptible to performance drift. In practice, one could handle abstentions by referring patients to human experts or ordering additional medical tests.

The problem of designing safe and effective pACPs for realworld settings is highly complex. We have studied the problem of distributional shifts within a simplified framework. Future works will need to extend our setup to more realistic settings. For instance, this work controls a univariate performance metric, but multiple metrics need to be tracked in practice (e.g. sensitivity and specificity, or performance across different subpopulations). In addition, we have assumed the monitoring data is representative of the target population and contains gold-standard labels. Obtaining such ideal data is not easy to do, and this sampling problem needs to be studied in much more detail. Finally, rather than full automation of the approval process, one may also consider pACPs that integrate human expertise and oversight.

\section{ACKNOWLEDGMENTS}

This work was greatly improved by helpful suggestions and feedback from Alexej Gossmann, Berkman Sahiner, Brian Williamson, Noah Simon, Pang Wei Koh, and Romain Pirracchio.

This publication was supported by the Food and Drug Administration (FDA) of the U.S. Department of Health and Human Services (HHS) as part of a financial assistance award Center of Excellence in Regulatory Science and Innovation grant to University of California, San Francisco (UCSF) and Stanford University, U01FD005978 totaling $\$ 79,250$ with 100 percent funded by FDA/HHS. The contents are those of the author(s) and do not necessarily represent the official views of, nor an endorsement, by FDA/HHS, or the U.S. Government.

Conflict of Interest: None declared.

\section{REFERENCES}

[1] Peter L Bartlett and Marten H Wegkamp. 2008. Classification with a Reject Option using a Hinge Loss. F. Mach. Learn. Res. 9, Aug (2008), 1823-1840. http: 
//www.jmlr.org/papers/volume9/bartlett08a/bartlett08a.pdf

[2] N. Cesa-Bianchi and G. Lugosi. 2006. Prediction, Learning, and Games. Cambridge University Press. https://books.google.com/books?id=zDnRBlazhfYC

[3] C K Chiang, T Yang, C J Lee, M Mahdavi, C J Lu, and others. 2012. Online optimization with gradual variations. Conference on Learning (2012). http: //www.jmlr.org/proceedings/papers/v23/chiang12/chiang12.pdf

[4] Alexandra Chouldechova and Aaron Roth. 2018. The Frontiers of Fairness in Machine Learning. (Oct. 2018). arXiv:1810.08810 [cs.LG] http://arxiv.org/abs/ 1810.08810

[5] Sharon E Davis, Robert A Greevy, Christopher Fonnesbeck, Thomas A Lasko, Colin G Walsh, and Michael E Matheny. 2019. A nonparametric updating method to correct clinical prediction model drift. 7. Am. Med. Inform. Assoc. 26, 12 (Dec. 2019), 1448-1457. http://dx.doi.org/10.1093/jamia/ocz127

[6] Sharon E Davis, Robert A Greevy, Thomas A Lasko, Colin G Walsh, and Michael E Matheny. 2019. Comparison of Prediction Model Performance Updating Protocols: Using a Data-Driven Testing Procedure to Guide Updating. AMIA Annu. Symp. Proc. 2019 (2019), 1002-1010. https://www.ncbi.nlm.nih.gov/pubmed/32308897

[7] Sharon E Davis, Thomas A Lasko, Guanhua Chen, Edward D Siew, and Michael E Matheny. 2017. Calibration drift in regression and machine learning models for acute kidney injury. F. Am. Med. Inform. Assoc. 24, 6 (Nov. 2017), 1052-1061. http://dx.doi.org/10.1093/jamia/ocx030

[8] Steven de Rooij, Tim van Erven, Peter D Grünwald, and Wouter M Koolen. 2014 Follow the Leader If You Can, Hedge If You Must. 7. Mach. Learn. Res. 15, 37 (2014), 1281-1316. https://jmlr.org/papers/v15/rooij14a.html

[9] Ran El-Yaniv and Yair Wiener. 2010. On the Foundations of Noise-free Selective Classification. F. Mach. Learn. Res. 11, May (2010), 1605-1641. http://www.jmlr org/papers/volume11/el-yaniv10a/el-yaniv10a.pdf

[10] FDA. 2019. US FDA Artificial Intelligence and Machine Learning Discussion Paper Technical Report.

[11] FDA. 2021. Artificial Intelligence/Machine Learning-Based Software as a Medical Device Action Plan. Technical Report.

[12] Jean Feng, Scott Emerson, and Noah Simon. 2020. Approval policies for modifications to Machine Learning-Based Software as a Medical Device: A study of bio-creep. Biometrics (2020)

[13] Jean Feng, Arjun Sondhi, Jessica Perry, and Noah Simon. 2019. Selective prediction-set models with coverage guarantees. arXiv (June 2019). http: //arxiv.org/abs/1906.05473

[14] Thomas R Fleming. 2008. Current issues in non-inferiority trials. Stat. Med. 27, 3 (Feb. 2008), 317-332. http://dx.doi.org/10.1002/sim.2855

[15] Marzyeh Ghassemi, Tristan Naumann, Peter Schulam, Andrew L Beam, Irene Y Chen, and Rajesh Ranganath. 2020. A Review of Challenges and Opportunities in Machine Learning for Health. AMIA ft Summits Transl Sci Proc 2020 (May 2020), 191-200. https://www.ncbi.nlm.nih.gov/pubmed/32477638

[16] Arthur Gretton, Karsten M Borgwardt, Malte J Rasch, Bernhard Schölkopf, and Alexander Smola. 2012. A Kernel Two-Sample Test. F. Mach. Learn. Res. 13, 25 (2012), 723-773. http://www.jmlr.org/beta/papers/v13/gretton12a.html

[17] Wei Han, Alexander Rakhlin, and Karthik Sridharan. 2013. Competing With Strategies. In Conference on Learning Theory. PMLR, 966-992. http://www.jmlr. org/proceedings/papers/v30/Han13.html

[18] Hrayr Harutyunyan, Hrant Khachatrian, David C Kale, Greg Ver Steeg, and Aram Galstyan. 2019. Multitask learning and benchmarking with clinical time series data. Sci Data 6, 1 (June 2019), 96. http://dx.doi.org/10.1038/s41597-019-0103-9

[19] Tatsunori Hashimoto, Megha Srivastava, Hongseok Namkoong, and Percy Liang. 2018. Fairness Without Demographics in Repeated Loss Minimization. In Proceedings of the 35th International Conference on Machine Learning (Proceedings of Machine Learning Research, Vol. 80), Jennifer Dy and Andreas Krause (Eds.). PMLR, Stockholmsmässan, Stockholm Sweden, 1929-1938 http://proceedings.mlr.press/v80/hashimoto18a.html

[20] Alistair Johnson, Lucas Bulgarelli, Tom Pollard, Steven Horng, Leo Anthony Celi, and Roger Mark. 2020. MIMIC-IV. https://physionet.org/content/mimiciv/0.4/

[21] Christopher J Kelly, Alan Karthikesalingam, Mustafa Suleyman, Greg Corrado, and Dominic King. 2019. Key challenges for delivering clinical impact with artificial intelligence. BMC Med. 17, 1 (Oct. 2019), 195. http://dx.doi.org/10.1186/ s12916-019-1426-2

[22] Jacqueline C Kirby, Peter Speltz, Luke V Rasmussen, Melissa Basford, Omri Gottesman, Peggy L Peissig, Jennifer A Pacheco, Gerard Tromp, Jyotishman Pathak, David S Carrell, Stephen B Ellis, Todd Lingren, Will K Thompson, Guergana Savova, Jonathan Haines, Dan M Roden, Paul A Harris, and Joshua C Denny. 2016. PheKB: a catalog and workflow for creating electronic phenotype algorithms for transportability. 7. Am. Med. Inform. Assoc. 23, 6 (Nov. 2016), 1046-1052. http://dx.doi.org/10.1093/jamia/ocv202

[23] Jeremy Z Kolter and Marcus A Maloof. 2005. Using Additive Expert Ensembles to Cope with Concept Drift. In Proceedings of the 22Nd International Conference on Machine Learning (Bonn, Germany) (ICML '05). ACM, New York, NY, USA, 449-456. https://doi.org/10.1145/1102351.1102408

[24] J Zico Kolter and Marcus A Maloof. 2007. Dynamic Weighted Majority: An Ensemble Method for Drifting Concepts. 7. Mach. Learn. Res. 8, Dec (2007), 2755-2790.
[25] Cecilia S Lee and Aaron Y Lee. 2020. Clinical applications of continual learning machine learning. The Lancet Digital Health 2, 6 (June 2020), e279-e281. https: //doi.org/10.1016/S2589-7500(20)30102-3

[26] Ron C Li, Steven M Asch, and Nigam H Shah. 2020. Developing a delivery science for artificial intelligence in healthcare. NPF Digit Med 3 (Aug. 2020), 107. http://dx.doi.org/10.1038/s41746-020-00318-y

[27] Katherine P Liao, Tianxi Cai, Guergana K Savova, Shawn N Murphy, Elizabeth W Karlson, Ashwin N Ananthakrishnan, Vivian S Gainer, Stanley Y Shaw, Zongqi Xia, Peter Szolovits, Susanne Churchill, and Isaac Kohane. 2015. Development of phenotype algorithms using electronic medical records and incorporating natural language processing. BMF 350 (April 2015), h1885. http://dx.doi.org/10. 1136/bmj.h1885

[28] Lydia T Liu, Sarah Dean, Esther Rolf, Max Simchowitz, and Moritz Hardt. 2018. Delayed Impact of Fair Machine Learning (Proceedings of Machine Learning Research, Vol. 80), Jennifer Dy and Andreas Krause (Eds.). PMLR, Stockholmsmässan, Stockholm Sweden, 3150-3158. http://proceedings.mlr.press/v80/liu18c.html

[29] Donald Martin, Jr., Vinodkumar Prabhakaran, Jill Kuhlberg, Andrew Smart, and William S Isaac. 2020. Participatory Problem Formulation for Fairer Machine Learning Through Community Based System Dynamics. (May 2020). arXiv:2005.07572 [cs.CY] http://arxiv.org/abs/2005.07572

[30] Lilian Minne, Saeid Eslami, Nicolette de Keizer, Evert de Jonge, Sophia E de Rooij, and Ameen Abu-Hanna. 2012. Effect of changes over time in the performance of a customized SAPS-II model on the quality of care assessment. Intensive Care Med. 38, 1 (Jan. 2012), 40-46. http://dx.doi.org/10.1007/s00134-011-2390-2

[31] Jaouad Mourtada and Odalric-Ambrym Maillard. 2017. Efficient tracking of a growing number of experts. International Conference on Algorithmic Learning Theory 76 (2017), 517-539. http://proceedings.mlr.press/v76/mourtada17a.html

[32] Bret Nestor, Matthew B A McDermott, Willie Boag, Gabriela Berner, Tristan Naumann, Michael C Hughes, Anna Goldenberg, and Marzyeh Ghassemi. 2019. Feature Robustness in Non-stationary Health Records: Caveats to Deployable Model Performance in Common Clinical Machine Learning Tasks. Machine Learning for Healthcare 106 (2019), 381-405. http://proceedings.mlr.press/v106/ nestor19a

[33] Ziad Obermeyer, Brian Powers, Christine Vogeli, and Sendhil Mullainathan. 2019. Dissecting racial bias in an algorithm used to manage the health of populations. Science 366, 6464 (Oct. 2019), 447-453. http://dx.doi.org/10.1126/science.aax2342

[34] Alexander Rakhlin and Karthik Sridharan. 2013. Online Learning with Predictable Sequences. jmlr.org, 993-1019. http://www.jmlr.org/proceedings/papers/v30/ Rakhlin13.html

[35] Alexander Rakhlin, Karthik Sridharan, and Ambuj Tewari. 2011. Online Learning: Stochastic, Constrained, and Smoothed Adversaries. In Advances in Neural Information Processing Systems 24, J Shawe-Taylor, R S Zemel, P L Bartlett, F Pereira, and K Q Weinberger (Eds.). Curran Associates, Inc., 17641772. http://papers.nips.cc/paper/4262-online-learning-stochastic- constrainedand-smoothed-adversaries.pdf

[36] Cosma Rohilla Shalizi, Abigail Z Jacobs, Kristina Lisa Klinkner, and Aaron Clauset. 2011. Adapting to Non-stationarity with Growing Expert Ensembles. (March 2011). arXiv:1103.0949 [stat.ML] http://arxiv.org/abs/1103.0949

[37] Yvonne Vergouwe, Daan Nieboer, Rianne Oostenbrink, Thomas P A Debray, Gordon D Murray, Michael W Kattan, Hendrik Koffijberg, Karel G M Moons, and Ewout W Steyerberg. 2017. A closed testing procedure to select an appropriate method for updating prediction models. Stat. Med. 36, 28 (Dec. 2017), 4529-4539. http://dx.doi.org/10.1002/sim.7179

[38] Yichi Zhang, Tianrun Cai, Sheng Yu, Kelly Cho, Chuan Hong, Jiehuan Sun, Jie Huang, Yuk-Lam Ho, Ashwin N Ananthakrishnan, Zongqi Xia, Stanley Y Shaw, Vivian Gainer, Victor Castro, Nicholas Link, Jacqueline Honerlaw, Sicong Huang, David Gagnon, Elizabeth W Karlson, Robert M Plenge, Peter Szolovits, Guergana Savova, Susanne Churchill, Christopher O’Donnell, Shawn N Murphy, J Michael Gaziano, Isaac Kohane, Tianxi Cai, and Katherine P Liao. 2019. Highthroughput phenotyping with electronic medical record data using a common semi-supervised approach (PheCAP). Nat. Protoc. 14, 12 (Dec. 2019), 3426-3444. http://dx.doi.org/10.1038/s41596-019-0227-6 